المياه

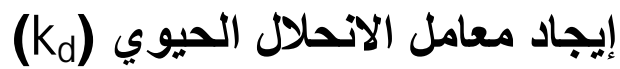

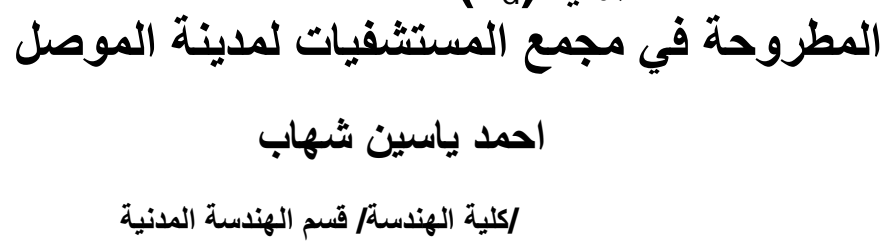

\title{
Determination of Bio-decay Coefficient of Disposed Activated Sludge from Wastewater Treatment Plant in Medical Assembly of Mosul City Ahmed Y. Shehab
}

University of MosulLEngineering CollegelCivil department

\begin{abstract}
This research, has dealt with determination of bio-decay coefficient of activated sludge disposed from wastewater treatment plant of medical assembly in Mosul city. four bench scale reactors were used of 2.25 liter capacity for each as aerobic digestion units. Throughout the operation period which lasted for a month temperature was controlled and it was $(15,20,25,30)$ in the first second third and fourth reactor respectively, the bio-decay coefficients was found as $\left(k_{d 15}=0.02, k_{d 20}=0.03, k_{d 25}=0.04\right.$, $\left.k_{d 30}=0.05\right) d^{-1}$, while the temperature constant $(\theta)$ was (1.059). Also the results showed that the required periods for aerobic digestion process to reduce $50 \%$ of biomass concentration in the reactors at digestion temperature $(15,20,25,30)$ were $(17,7,5,4)$ days respectively.
\end{abstract}

Key words: biological treatment, aerobic digestion, sludge disposal, bio-decay coefficients 
الهاضم (digestion unit) من الوحدات المهمة في محطات معالجة المياه

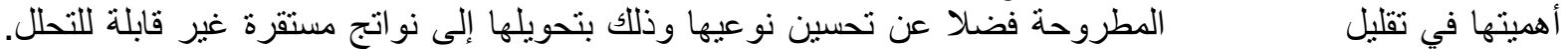

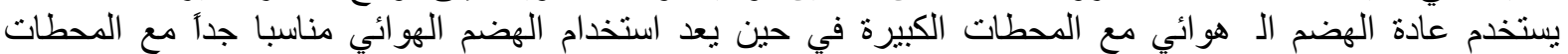

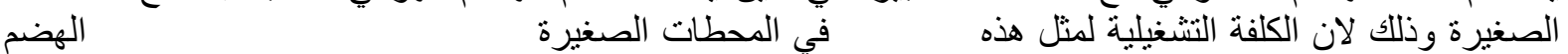

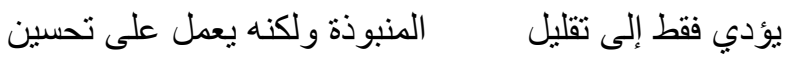
هوائي

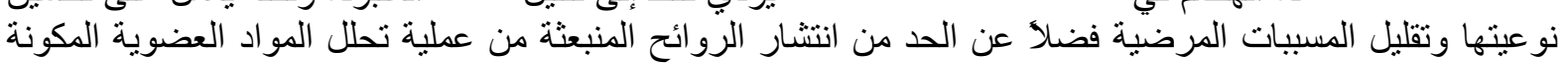

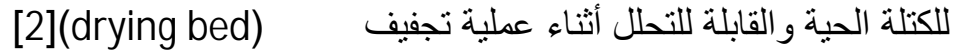

الضرورية لتصميم حوض الهضم الهوائي حيث يعد تحديد قيم معامل الانحلال الحيوي تختلف قيم هذا المعامل تبعأ لعدة عو امل أهمها نوعية الفضلات التي تتغذى عليها الأحباء المجهرية المكونة لتلكي

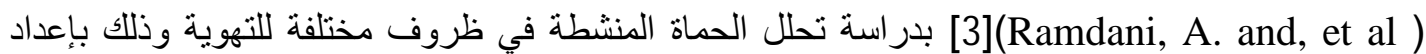

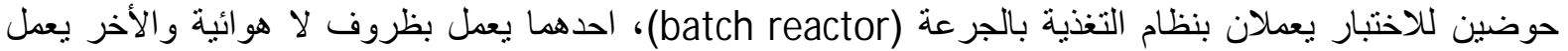

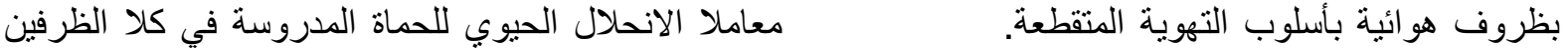
بأنه لتخفيض نركيز الحماة المهضومة في كلادة بنسبة حيث كانا

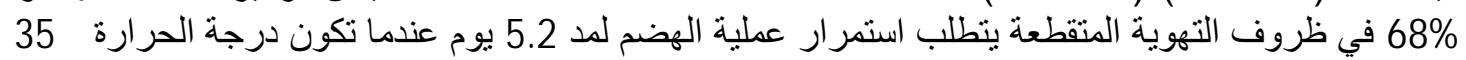
[4] (4ike H. Kim; and Oliver J. Hao) مع هضمها بظروف متعادلة للتهوية (anoxic conditions ).

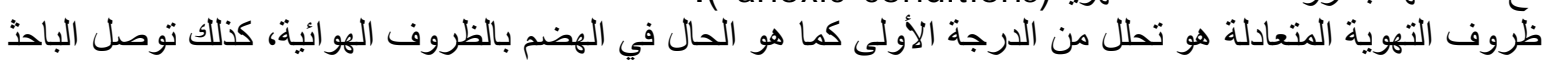

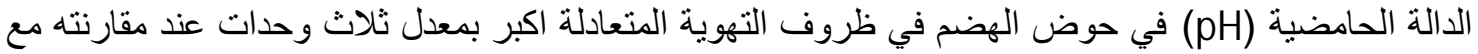
بسبب الاختلاف بسعة القاعدية(buffering capacity) بين الحالتين.

(5roste, R. L; and Sanchez, W. A) (10, 20, 30 C )

إلى انه لا

بالجرعة والثاني يعمل بأسلوب التغذية المتقطعة (10) بونة (semi continuous flow patterns) علاقة واضحة يمكن من خلالها وصف سر بلغة عة تحلل الحماة عند استخدم أسلوب التغذية المتقطعة.

[6] بدراسة لهضم الحماة المنشطة الناتجة من معالجة المطروحات (Bernard, S.; and Gray, N. F)

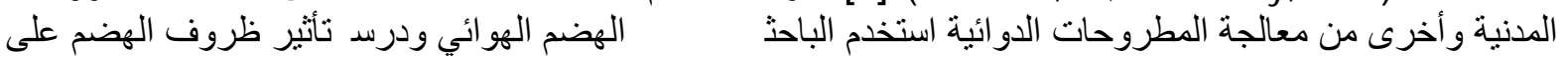
خصائص الحماة المهضومة.

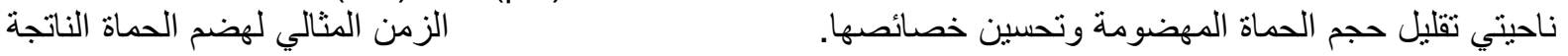

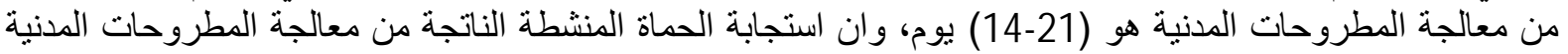
نسبة المواد المتطايرة بحدود المنات

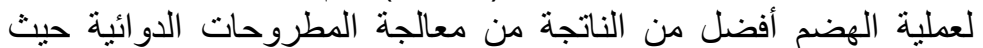
$(6 \%-25) \% \quad(53-64) \%$ 


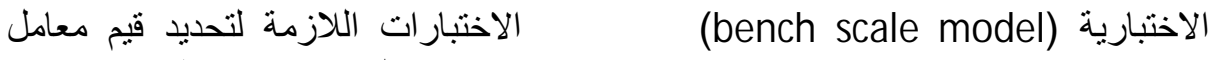

، حيث يتم هضم هذه الحما ل

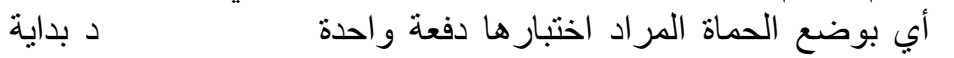

الحيوي (kdic (lic

(aerobic digest)

التشغيل (batch flow condition)
(1)

هو اء حجرية

بضاغطة هواء (compressor)

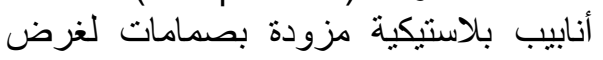

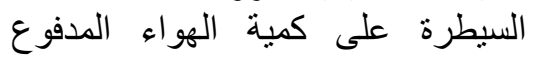

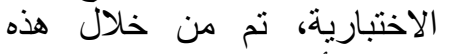

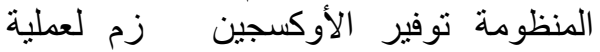

هضم المركبات العضوية الداخلة في

تركيب الأحياء المجهرية والقابلة للتحلل

يعمل الهواء المندفع عبر

عملية مزج تام عام

$$
\text { لمخته }
$$

ولغرض السيطرة على درجة الحرارة

$$
\text { في الأحواض الاضحرية فيبارية }
$$

(mesophilic bacteria)

تزويد الأحواض بسخانات كهربائية ذات

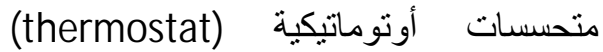
لة الحرارة ضمن المستويات$$
\text { مختبريه اسطو اني }
$$

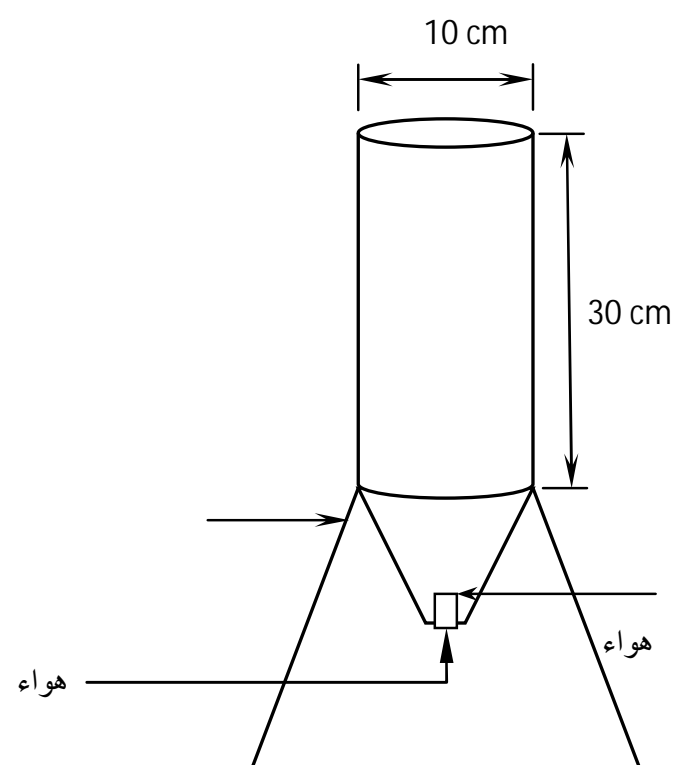

$:(1)$

$$
\text { [8] حيث كانت درجة الحرارة في الحوض الأول ( 15) في حين }
$$

تم انجاز البحث خلال الفترة الممتدة بين (2009/02/01 2009/03/11) في مختبر هندسة البيئة في كلية

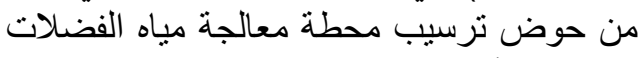

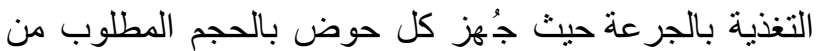

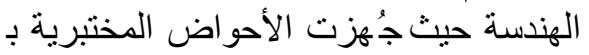

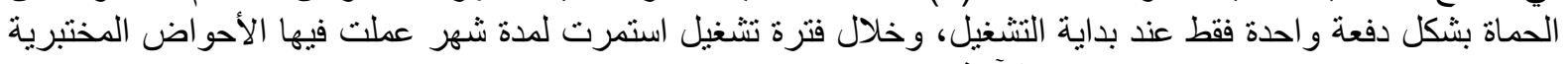
$:[9]$

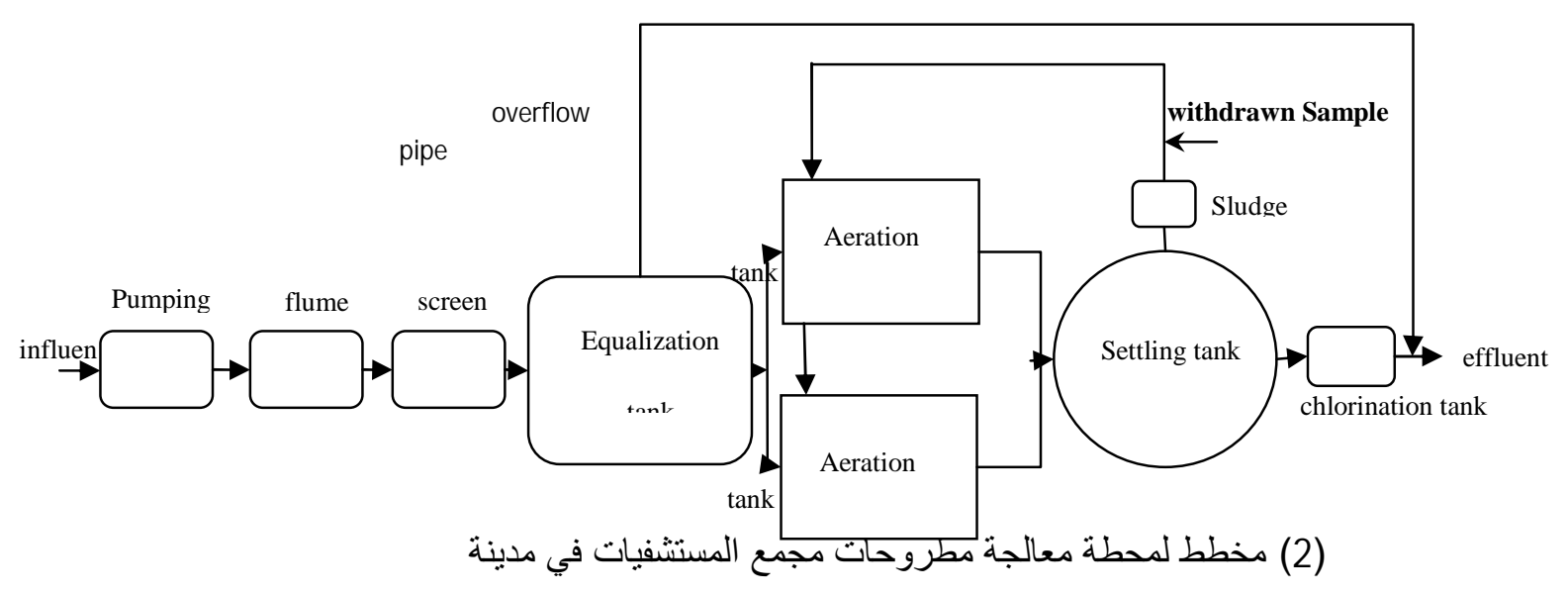


1. قياس تركيز المواد الصلبة العالقة التطايرة(M LVSS): تم هذا الفحص بشكل متواصل وبمعدل قراءة

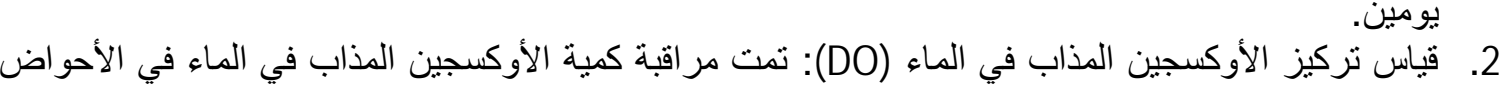

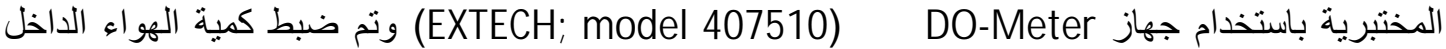

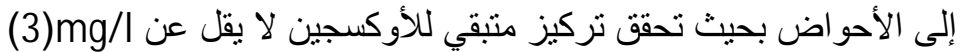

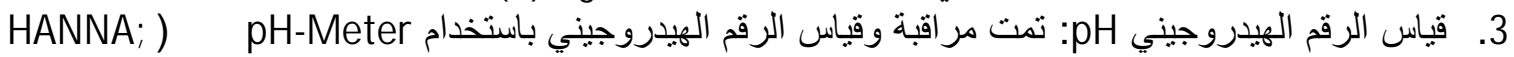

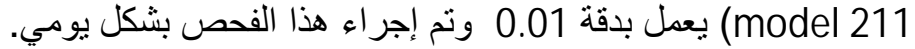

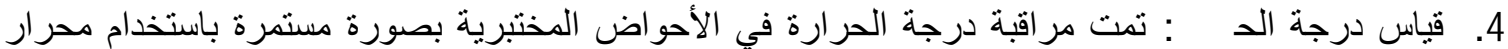
زئقي يعمل بالنظام المئوي بدقة 0.5 درجة مئوية

$$
\begin{aligned}
& \text { المهضومة ويت تركيز الأحياء المجهرية } \\
& \text { [7] } \\
& \text { (first order kinetics) } \\
& \frac{d x}{d t}=k \cdot x \\
& \frac{\left(x_{d}\right)_{e}}{\left(x_{d}\right)_{o}}=e^{-t k_{d}}
\end{aligned}
$$

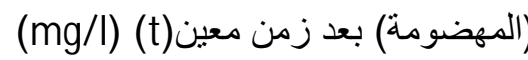

$$
\begin{aligned}
& \text { 㳊 } \\
& \text { (mg/l/ (mo ) : التركيز } \\
& \text { (d : معل الانحلال الحيوي للحماة المهضومة : } \\
& \text { (d) التهن : : }
\end{aligned}
$$

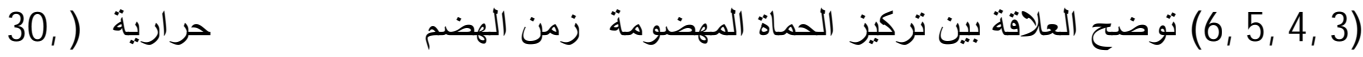

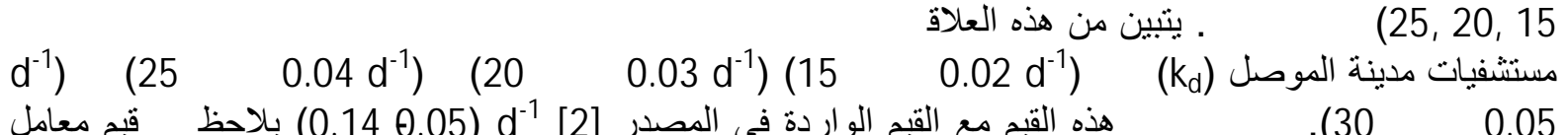

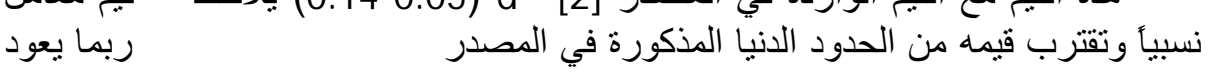

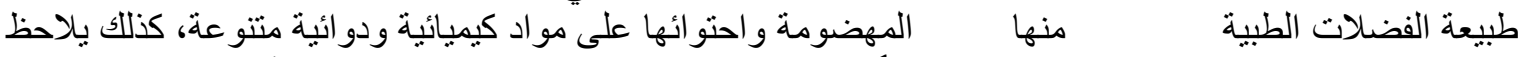

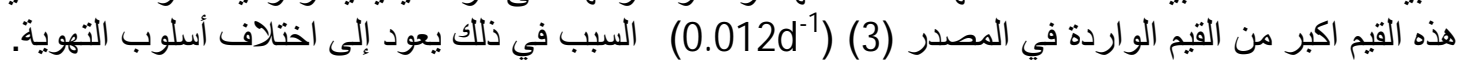

يتيبن من الأشكال انه عند تشغيل أحواض الهضم الاختبارية لفترة زمنية تساوي (30,250, (30) يومأ كانت النسبة

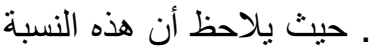

المئوية للمواد العضوية المتحللة \% (84)

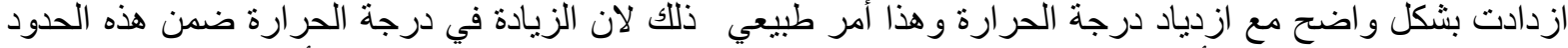

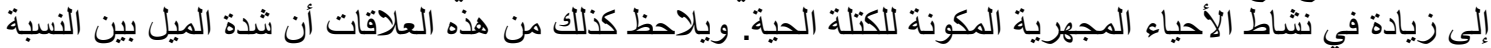

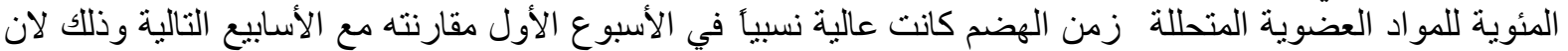

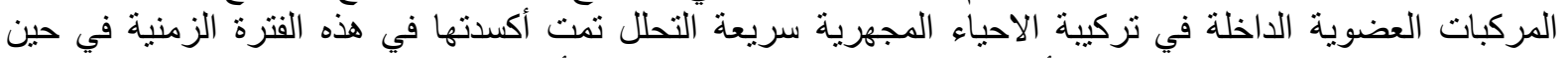

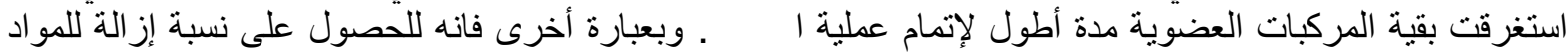

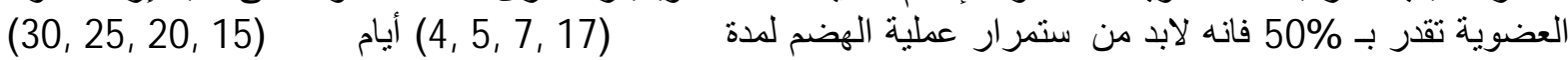

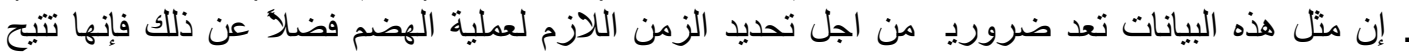

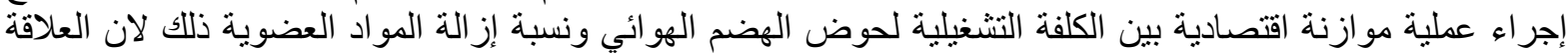
بين هذين العاملين ليد علاقة خطية. 


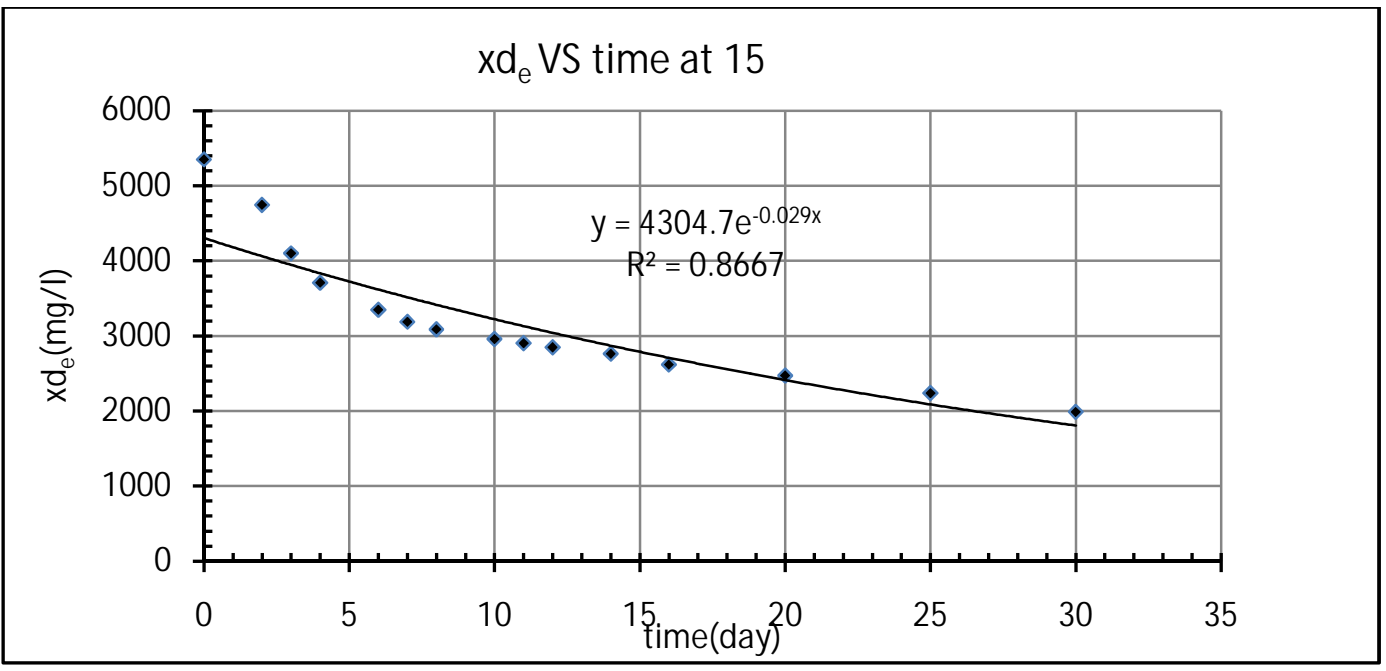

(3): العلاقة بين تركيز الأحياء المجهرية المتحللة وزمن الهضم عند درجة حرارة ( 15 )

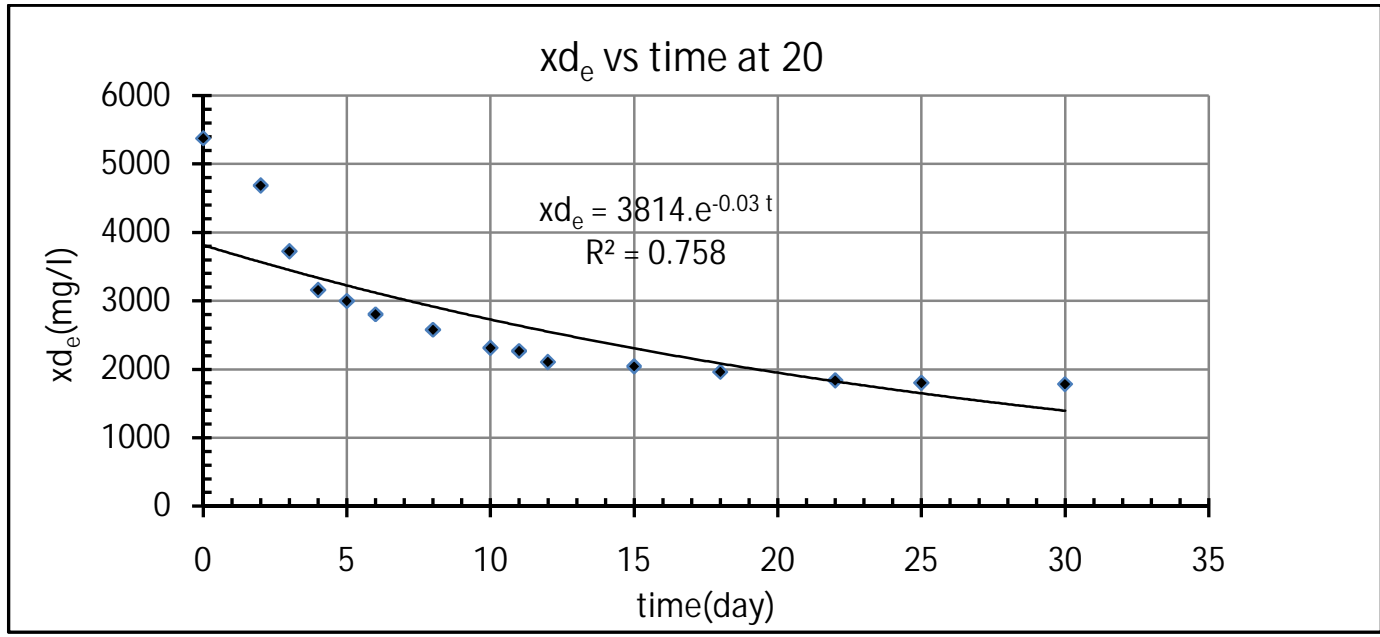

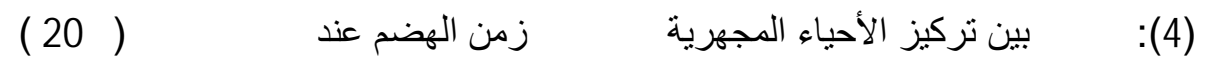

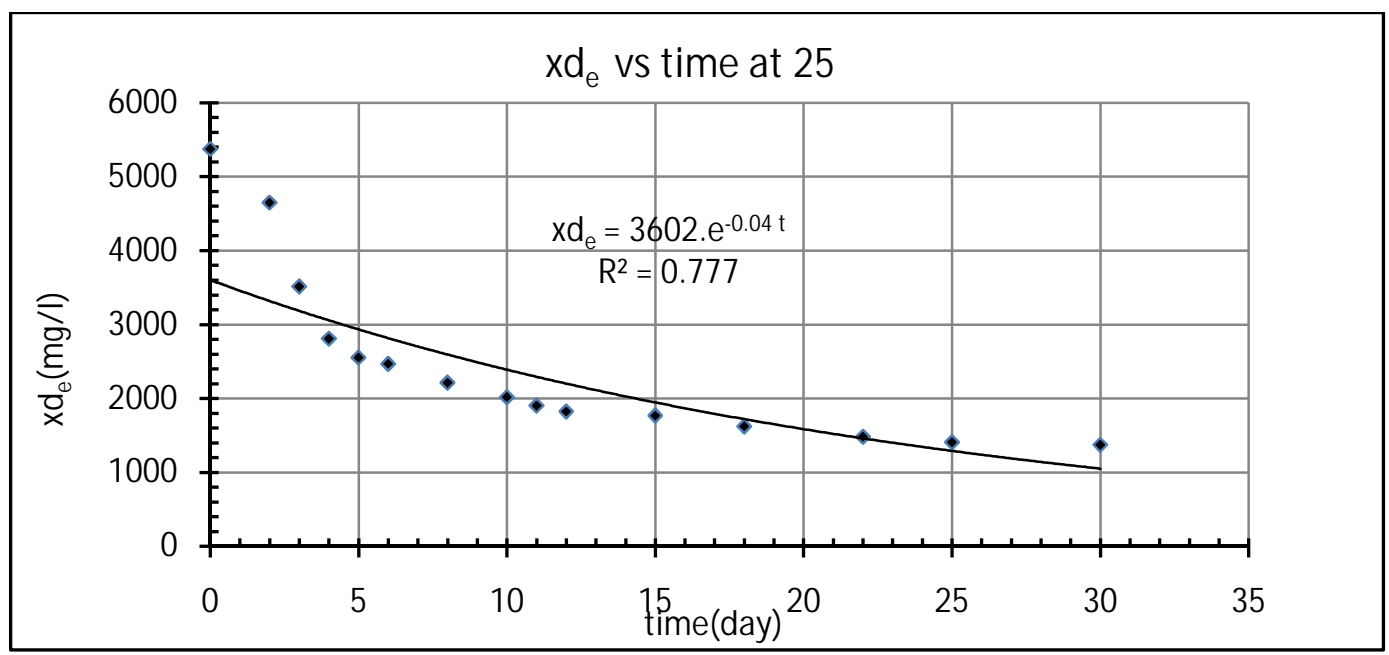

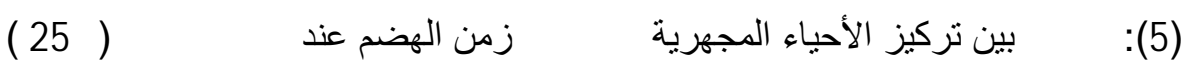




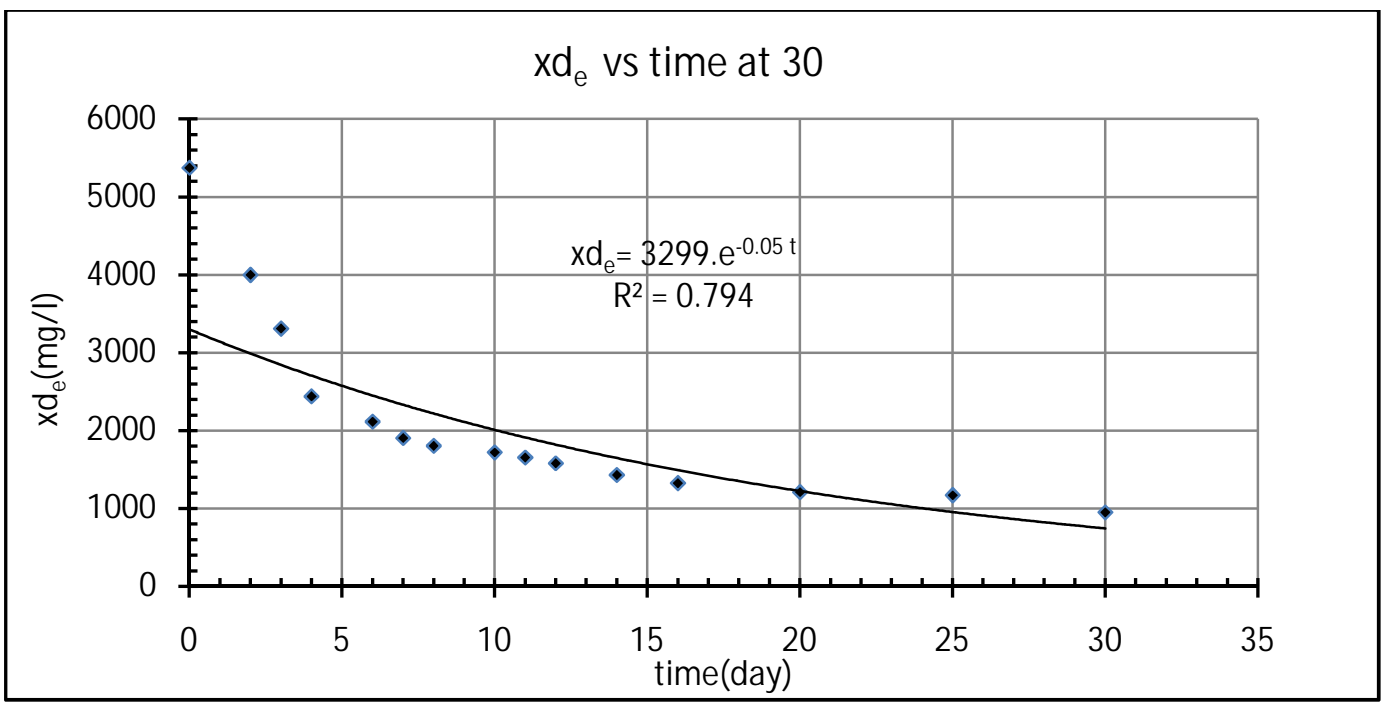

(30)

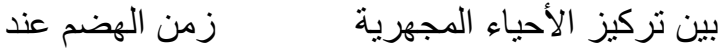

و وعند اختبار فرضية الحماة المهضومة؛ تتحلل هو ائيا بموجب معادلة من الدرجة الثانية ( second order

(reaction

$$
\frac{d x}{d t}=k \cdot x^{2}
$$

$$
\frac{1}{\left(x_{d}\right)_{e}}-\frac{1}{\left(x_{d}\right)_{o}}=\mathrm{k}_{\mathrm{d}} \cdot t
$$

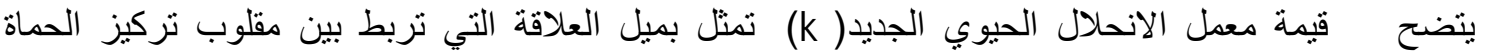
المهضومة عند زمن معين وقيمة زمن الهضم وكما موضح في

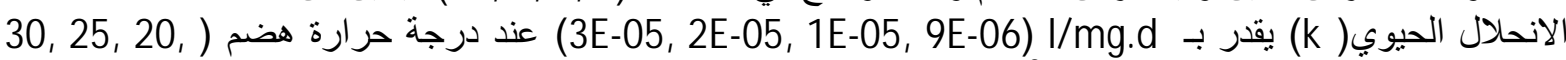
(R²)

\section{$1 / X_{e}$ VSt at $15 \dot{c}$}

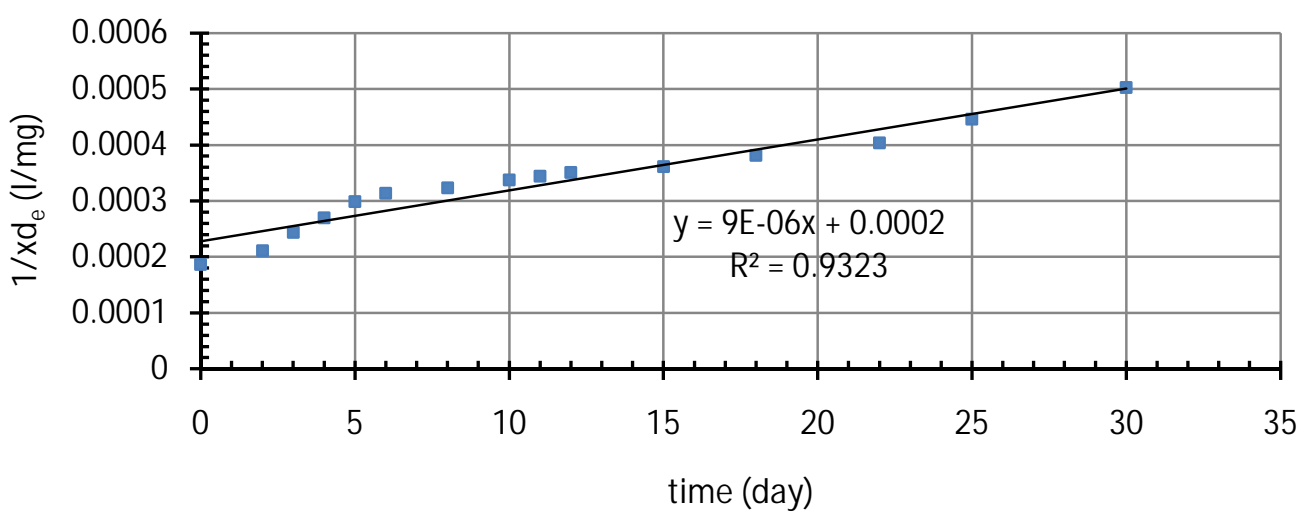

(7) العلاقة بين مقلوب تركيز الحماة وزمن الهضم عند درجة حر ارة ( 15 ) 


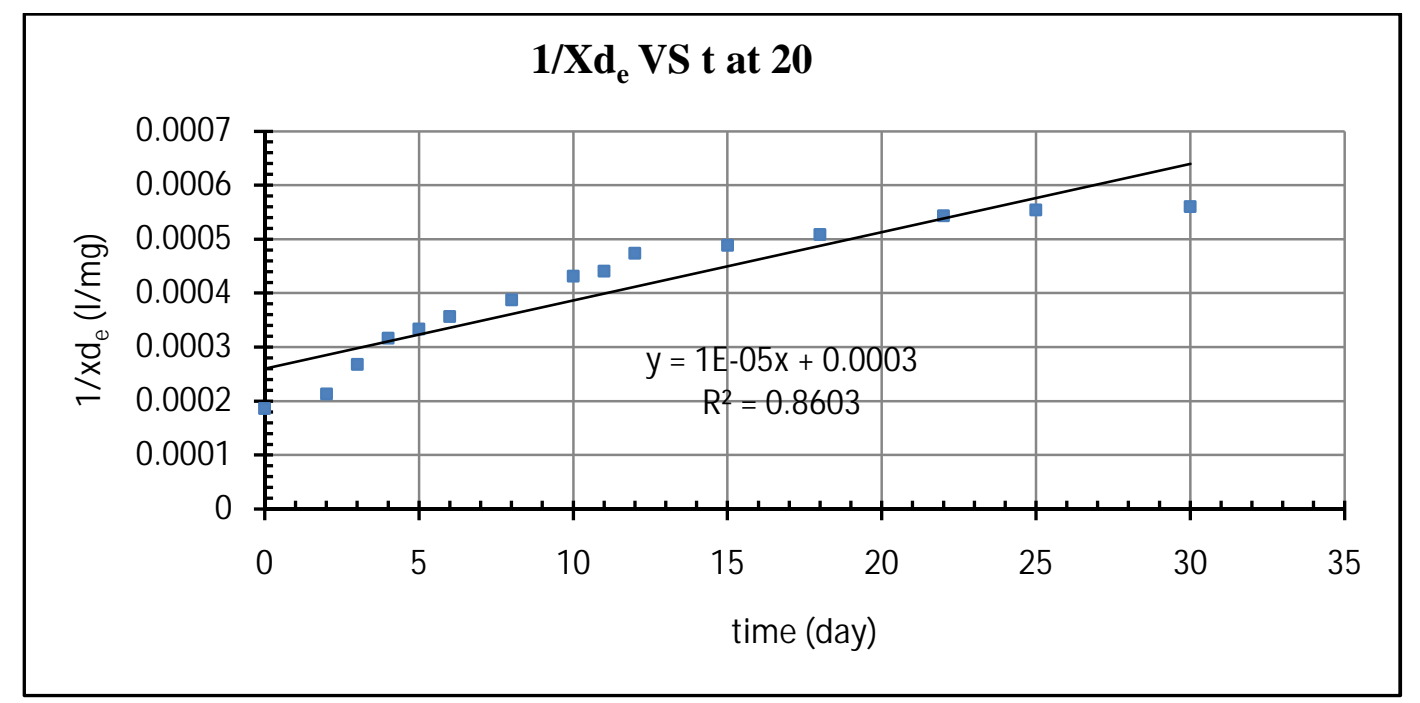

(8) العلاقة بين مقلوب نركيز الحماة وزمن الهضم عند درجة حرارة ( 20)

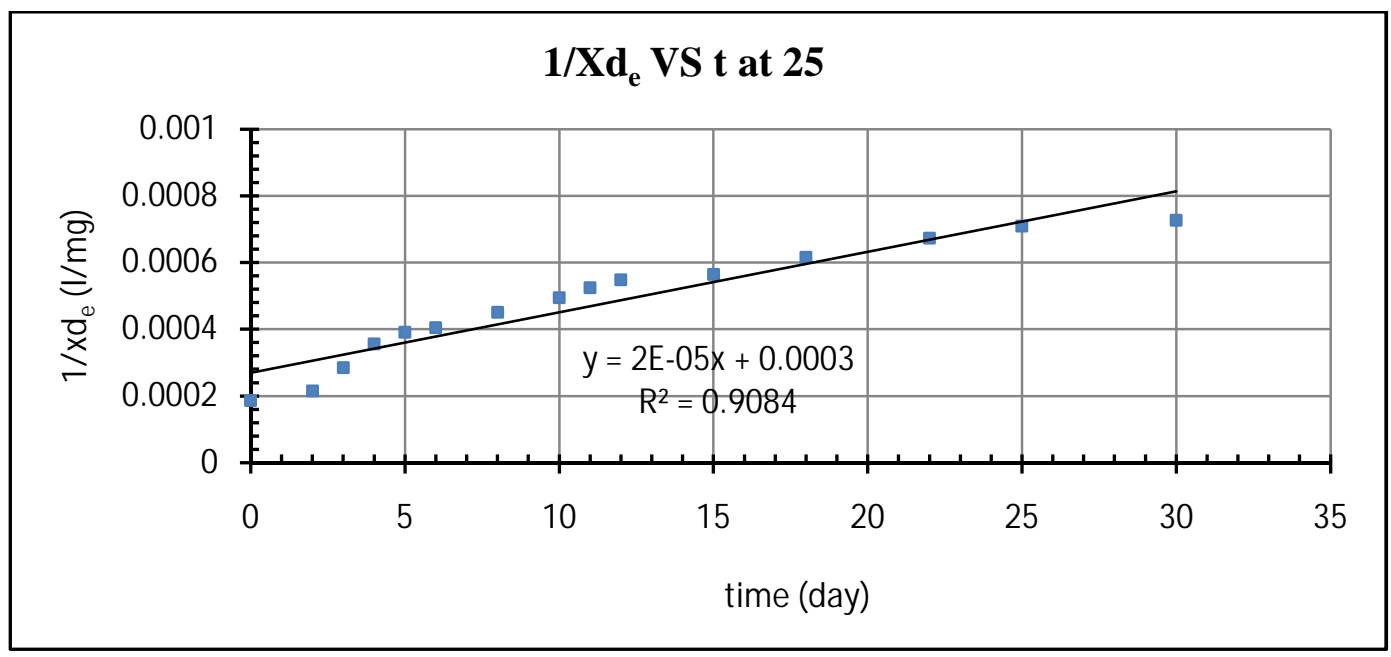

(9) العلاقة بين مقلوب تركيز الحماة وزمن الهضم عند درجة حرارة ( 25 )

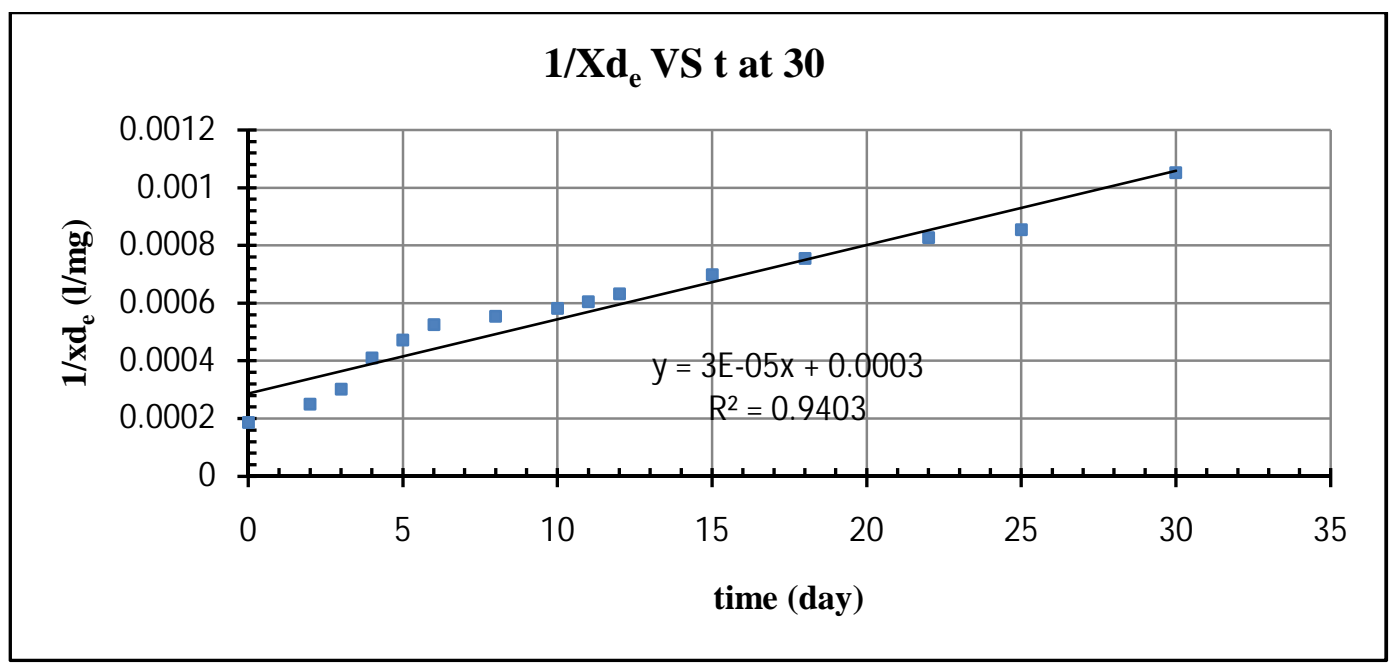




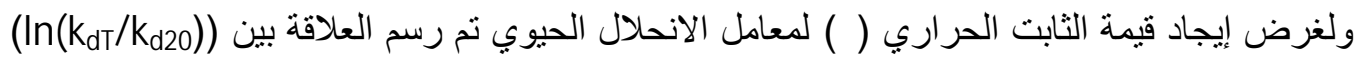

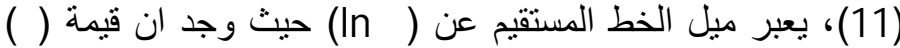

هذه القيمة مع الحدود الواردة في المصدر [7] (1) (1.04) وجد بأنها

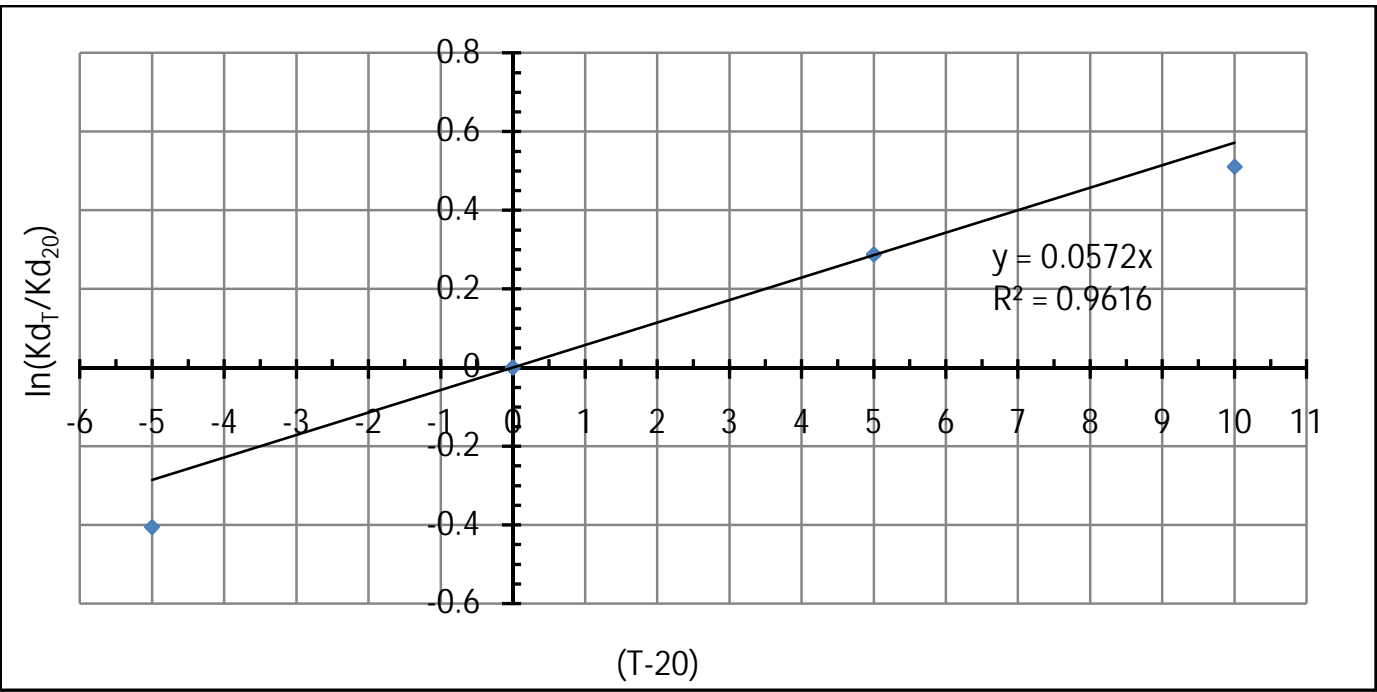

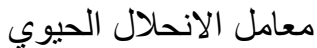

(11)

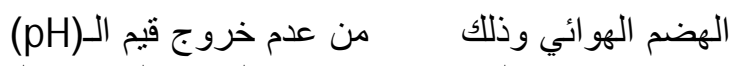

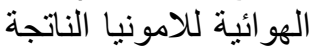

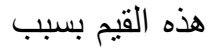

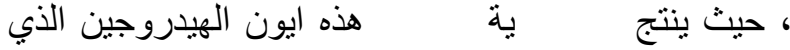

ومن الضروري مر اقبة الدالة الحامضية في

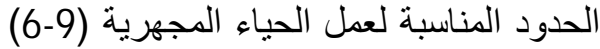

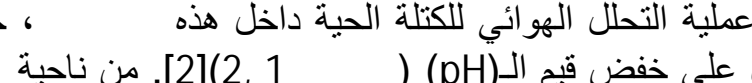
بها (pH) سوف تنخفض قيم (p)

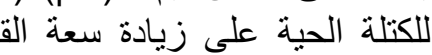

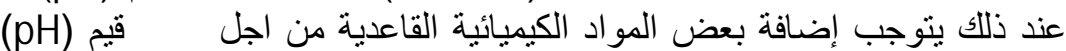

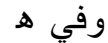

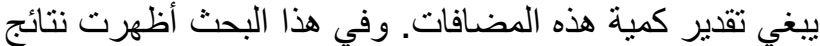

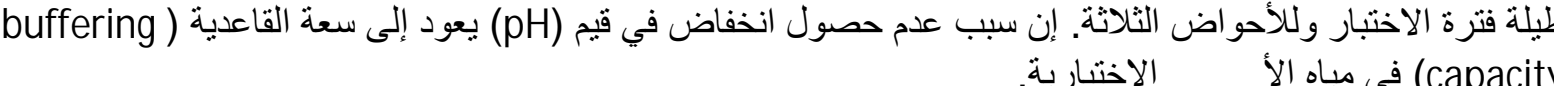

$\mathrm{C}_{5} \mathrm{H}_{7} \mathrm{NO}_{2}+5 \mathrm{O}_{2} \rightarrow 4 \mathrm{CO}_{2}+\mathrm{H}_{2} \mathrm{O}+\mathrm{NH}_{4} \mathrm{HCO}_{3}$

$\mathrm{NH}_{4}^{+}+2 \mathrm{O}_{2} \rightarrow \mathrm{NO}_{3}+2 \mathrm{H}^{+}+\mathrm{H}_{2} \mathrm{O}$

$\mathrm{CO}_{2}+\mathrm{H}_{2} \mathrm{O} \leftrightarrow \mathrm{H}_{2} \mathrm{CO}_{3} \leftrightarrow \mathrm{H}^{+}+\mathrm{HCO}_{3}^{-}$

$\mathrm{CO}_{2}+\mathrm{OH}^{-} \leftrightarrow \mathrm{HCO}_{3}^{-} \leftrightarrow \mathrm{H}^{+}+\mathrm{CO}_{3}^{2-}$ 
1. Cheremisinoff, N.P. (2002) "Handbook of water and wastewater treatment technologies", Butterworth-Heinemann, USA.

2. Metcalf and Eddy, Inc. (2003) "Wastewater engineering treatment/disposal /reuse", fourth edition, McGraw-Hill, Inc, New York.

3. Ramdani, A. Dold, P.; Déléris, S.; Lamarre, D.; Gadbois, A.; and Comeau, Y. (2007) " Biodegradation of the endogenous residue of activated sludge", Journal of Water Research, Vol.47, Issue 68

4. Mike H. Kim; and Oliver J. Hao (1990) "Comparison of Activated Sludge Stabilization under Aerobic or Anoxic Conditions", Journal of the Water Pollution Control Federation, Vol. 62, No. 2, pp. 160-168

5. Droste, R. L; and Sanchez, W. A. (2004) "Modeling Active Mass in Aerobic Sludge Digestion", Journal of Biotechnology and Bioengineering, Vol. 28, Issue 11, Pages 1699 $-1706$

6. Bernard, S.; and Gray, N. F (2000) "Aerobic Digestion of Pharmaceutical and Domestic Wastewater Sludges at ambient temperature ", Journal of Water Research, Vol. 34, Issue 3, Pages 725-734

7. Eckenfelder, W.W. (2000) "Industrial Water Pollution Control", McGraw-Hill, New York, third edition.

8. Fouad, M. and Bhargava, R. (2005) "Sludge Production and Settleability BiofilmActivated Sludge Process", Journal of Environmental Engineering, Vol. 131, No. 3. pp.417-424

9. APHA; AWWA; WPCF (1998) "Standard methods for the examination of water and wastewater", $20^{\text {th }}$ ed., Am. Public Healthy Assoc. Washington, D.C., USA

10.Viessman, W.Jr. and Hammer, M.J. (1985) "Water Supply and Pollution Control", Harper and row Publishers Inc., New York, fourth edition.

تم اجراء البحث في كلية ألهندسة = 